\title{
Utilizing real-time market demand information in the integration of the supply chain in the food industry
}

\author{
J.Slotte, M.Sc.(Eng.) \\ E-mail: Jonas.Slotte@hut.fi \\ H.Nyman, M.Sc.(Eng.), M.Sc.(Econ.) \\ E-mail: Hannu.Nyman@hut.fi \\ J.Holmström, Ph.D.(Eng.)
}

Helsinki University of Technology, TAI Research Center P.O.BOX 9500, FIN-02150 HUT, Finland

Tel. +358-9-4511, Fax +358-9-4513665

\begin{abstract}
This paper outlines exploitation of the existing communication infrastructure to improve the interaction between various echelons of the logistic chain in the food processing industry, right from the mill to the table of the consumer. It is commonly known that step-wise delivery chains tend to accumulate the true demand at the upstream of the chain, i.e. rather stable demand pattern is amplified along the chain resulting a significant demand variation at the factory. Key issues are related on how to collect reliable real-time information of the end consumption and on how to distribute it to all players of the logistic network. Other important facets of the research concern electronic communication and how to establish an information integrated supply chain.
\end{abstract}

\section{Keywords}

Diverging logistic network, demand amplification, electronic communication, information integrated supply chain, food-processing industry 


\section{INTRODUCTION}

A modern production control system requires input not only from production and material requirements planning, but also from various points in the whole supply chain. The customer point of view was introduced in manufacturing with the JustIn-Time philosophy. In a competitive customer oriented manufacturing environment the questions of customer responsiveness is the main focus. Increasingly market surveys find that customers see the delivery performance and accuracy as the most important feature of the supplier. For example, A.T. Kearney (1993) found in a survey commissioned by the European Logistics association a wide gap between supplier delivery service and the service levels required by customers.

In the traditional MRP literature, focus is put on production lead-time in the search for a fast reacting and lean operation. If the total production lead-time, including the planning lead-time of the products is longer than the required delivery time to customer the operation has to rely on demand forecasts (Stalk and Hout 1990). The more production is dependent on forecasts the more customer service has to rely on goods in stock.

In a modern operation, Quick Response Capability and Efficient Consumer Response (ECR) are the focus in supply chain and supplier management. Quick response capability is the ability to deliver the full product range within the customer acceptable delivery time. In the evaluation of the quick response capability of a production and distribution system, the focus must be put simultaneously on time and quantity. The lead time to produce the full product range is compared to the customer acceptable delivery time. At the same time customer demand is compared to capacity within the customer acceptable delivery time.

ECR on the other hand is a new philosophy in product range management and co-operation between supplier and wholesaler in the commodities market.

\section{SUPPLY CHAIN DYNAMICS AND CONTROL}

The control practices of industrial firms directly affect how the operational situation is perceived and how effectively resources are allocated.

Uncertainty of the operational situation and the controllability of the operation are emergent properties of the operational practices of the firm and the industries it is engaged in. This view is based on the observations that:

- demand changes are easily distorted and amplified in supply chains (Forrester, 1961)

- unsynchronized planning and control practices induce load surges (Burbidge, 1989 and 1994)

Thus, operational uncertainty and inefficiency is to a large extent a consequence of demand distortion in supply chains, self-induced uncertainty caused by unsynchronized planning. Demand distortion was first described by Forrester in his 
classic supply chain model, but it is only recently that the full extent of the problem has been recognized (Towill, 1996). Congestion caused by quality problems and rework, and complexity imposed by product designs and processes are other important contributing factors. Towill (1992) presents an example of how the combined effect of demand amplification in supply chains and load surges caused by unsynchronized control procedures results in high uncertainty of the operational situation.

The basic principle proposed to reduce self-inflicted operational uncertainty and to enable a more efficient use of resources is to focus efforts on speed and accuracy of communication and control of the information available in the supply chain.

The accumulation of needs in supply chains is discussed as a basic cause for operational uncertainty and low controllability. The purpose is to demonstrate that high operational uncertainty and low controllability are partly self-induced and may be avoided by implementing improved communication and control practices in the management of the supply chain. The discussion is based on experiences from development projects in the food industry.

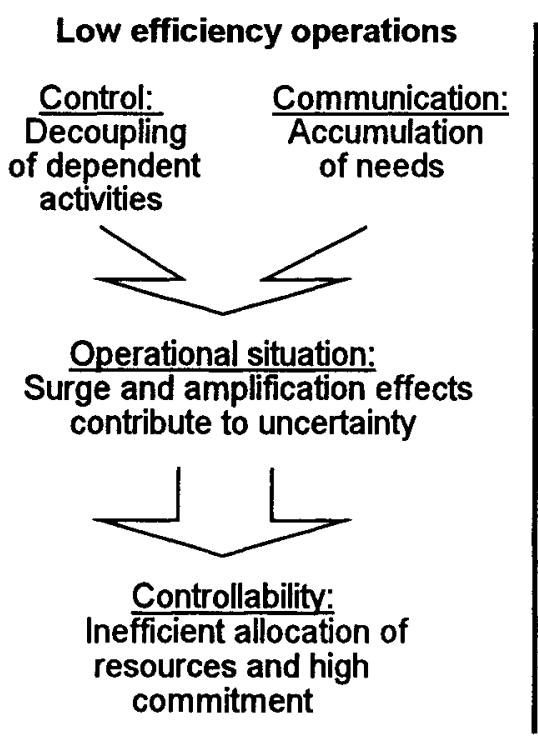

\section{High efficiency operations}

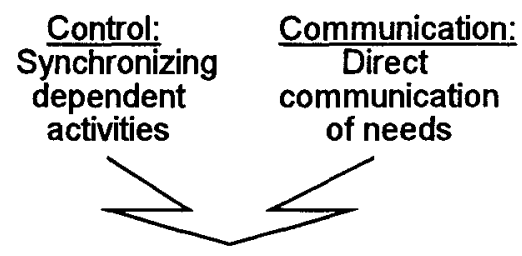

Operational situation:

Clarity and overview reduce uncertainty

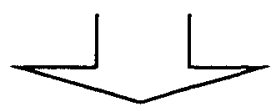

Controllability:

Efficient allocation of resources and low commitment

Figure 1 Interdependency of communication, uncertainty, and controllability.

\subsection{The effect of demand amplification in the supply chain}

The effect of accumulation is clearly evident in the operation of the sweet manufacturer case. The sweets manufacturer has both manufacturing and distribution operations in all of the Scandinavian countries and Finland.

The manufacturer perceives that demand for its products are highly volatile and unpredictable. The deliveries from the largest factory of a selection of key products 
were analyzed. The analyzed products are high volume products representing roughly $50 \%$ of the total output of the factory. In the delivery volumes significant medium-term fluctuations were present. For example, over a half year period there was first a dramatic surge followed by a sharp decline of demand. In the short-term the manufacturer experienced delivery problems due to great demand variations from week to week. However, over the long term consumption had been rising but only slowly. From week to week the consumption of sweets was stable, hence, this is an example of amplification caused by an accumulation of needs in the distribution chain.

The conclusion of the analysis was that the sweets manufacturer supplies an oscillating distribution chain. Pipeline inventory accumulation alternates with the distribution chain living off accumulated inventory buffers. This oscillation was clearly visible when comparing the delivery and consumption of the main product (Figure 2). Deliveries were first lower than consumption, then higher than consumption, and finally below consumption again.

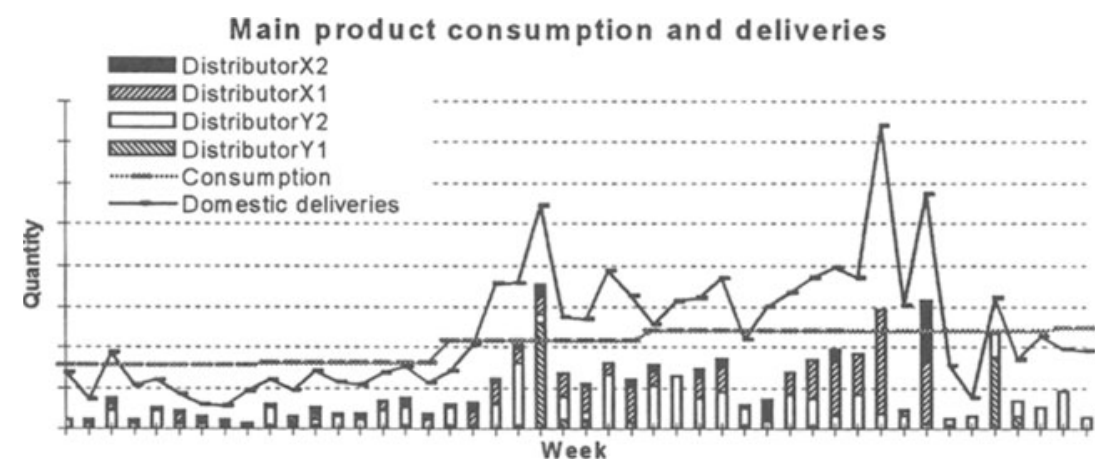

Figure 2 Example of amplification caused by accumulation of needs

The unpredictable delivery peaks caused havoc for the sweets manufacturer because the distribution network responded to signs of weakening delivery capabilities by increasing buffers. The perceived radical rise in demand prompted investment in more production capacity for the manufacture of the main product. Additionally, resources were wasted on training new employees who later proved unnecessary. Overtime and extra shifts were also necessary to meet the peak demand. In the subsequent quiet periods it was necessary to close production lines for a week on several occasions.

In the sweets manufacturer case it is obviously extremely difficult to run the operation efficiently over a longer period of time as long as the oscillating supply chain distorts demand. 


\subsection{Required delivery time}

In the consumer goods market a special feature is the required delivery time to the wholesalers. In Finland and Scandinavia it is from four to $\mathbf{4 8}$ hours. In an industry where the manufacturers product range can be up to 500 , the whole product range cannot be manufactured in the required delivery time. The solution to this is delivery from stock. In an operation there are three possibilities to control the deliveries of manufactured goods:

- The full product range can be planned, produced and transported within the customer acceptable delivery time.

- The delivery time requirements from customers are shorter than the time to plan and produce the full product range and to transport it to the customers.

- An intermediate point in the production process can be found from which onwards the full product range can be planned, produced and transported within the customer acceptable delivery time.

The demand of consumer goods and especially food products are quite stable in all the examples analyzed. The case examples cover meat, confectionery, dairies and brewery industries. The only exceptional demand pattern is in the brewery industry, where warm periods in the summer time affect demand dramatically.

\subsection{Market demand and Efficient Consumer Response}

The industry's answer to the need of more effective management practices is to improve its operating efficiency and service (Pontin et al. 1995). This effort is known as Efficient Consumer Response ( e.g. Robins, 1994, Yohalem, 1995). The goal is to eliminate waste and increase service by building up a demand driven supply chain. The focus of both suppliers and retail chains is to improve profitability and market share by responding better and quicker to consumer demand. In the retail trade the roles of product brands and product line extensions are diminishing and the importance of strategic product categories is rising (Quelch and Kenny, 1994). The essence of this shift of focus is to align service with consumer needs.

\section{UTILIZING REAL-TIME DEMAND INFORMATION}

The existing operations in the food industry can be improved by using real-time demand information. This information can be used in the production planning to shorten the delay between actual selling of the goods and the arrival of the order to the supplier. If the supplier gets accurate information about sales earlier he can plan his production more optimally.

The use of this technology is based on the assumption that the supermarkets belong to a chain controlled by a wholesaler, like K-Mart in the USA. If several such chains make up a significant part of the consumer market, their combined demand information is good enough to be used as an estimate for the whole market. 


\subsection{Using product sales information}

The key in this process is the utilization of information provided by bar codes in the actual products. The bar codes enable us to collect information about sales of each product. The sales information is collected by the cash registers in the shops. The information collected by cash registers in a shop is first combined on the shop level and then transmitted into the wholesaler. In the test cases in the Finnish consumer goods market this process is done daily. Thus the wholesaler gets accurate sales information from the shops and gets a clear picture of each product's actual sales almost in real-time.

This is already part of the normal daily operations. But this sales information has value also to the other parties in the logistics chain. The suppliers could use it for their production planning and their material suppliers could use the production schedules based on the acquired sales information. The main idea is to shorten the delay between the actual sale and the moment when the supplier gets the order to refill wholesalers storage.

The wholesaler, who is the customer of the product supplier, accumulates the combined sales figures for those products that belong to their product range. This information can be processed so that the weekly demand of products of a supplier is calculated and transmitted to the supplier.

The wholesaler can also give information about his product inventory. The supplier could use also this information to enhance production planning. The production planning can be based on this ideology only in those cases where the wholesaler tries to keep the inventory levels constant on the long term. If the wholesaler's inventory has no clear correlation to the sales, the inventory information does not provide any new information of use.

\subsection{The benefits of the information exchange}

This exchange of sales information is not meant to replace the normal ordering process. The main idea is to use sales information as an early warning of forthcoming orders.

\section{Wholesaler and suppliers}

Accurate sales information can increase the delivery performance since the supplier gets information about product consumption earlier. He can then plan his production based on the assumption that the shops will refill their inventory from the wholesalers who in turn then will refill their own storage by ordering the products from the supplier. The time between the actual sales and the arrival of the responding order is usually several weeks. On the other hand the allowed delivery time is currently very short, only 24 to 48 hours. Because the production lead times are remarkably longer the suppliers are forced to keep large inventories in order to be able to fulfill the orders in time. If the supplier gets the sales information shortly 
after the sales, e.g. on weekly basis, he can plan his production based on this information and be better prepared to fulfill the arriving orders.

So, the idea is to give supplier more time to plan his production. He does not need to wait for the actual order but he can start the production planning shortly after the actual sales based on reliable information. The delivery performance should increase since obtaining information earlier diminishes the effects of long lead times.

Another aspect to be considered is the quality of the information. The aggregate weekly consumption figure does not suffer from the Forrester effect or Burbidge effect. This figure is not biased in any direction and the different order periods and ordering strategies do not effect the figures. So, this is a much better data to be used for production planning than the figure obtained from arriving orders.

This holds true in the long term. In long term this will enable the supplier to fulfill the orders and to keep the product inventories on a reasonable level. In the short term, the supplier's inventory will fluctuate since the arriving orders do not equal the actual consumption since the ordered product volumes are distorted by Forrester and Burbidge effects.

\section{Supplier and raw material suppliers}

If the supplier has a tight relationship with his key material suppliers, the supplier can provide this sales information further to the material suppliers. That makes it easier for them to be able to deliver all required raw materials in time. In order to be able to benefit from the sales figures the material suppliers have to know roughly the raw material requirements of products.

Another way to deliver quality information to the material suppliers is to keep them aware of the planned production. If they know the material requirements and the production schedules they can calculate the actual material requirements.

Both methods give a qualified material requirements estimate to the material supplier. This estimate can be used in the production scheduling process before the formal order from the client arrives.

\subsection{Requirements for the co-operation}

The previously explained co-operative communication sets many requirements for the participants. The co-operation can only be realized in a stable and continuous business relationship. The participating companies must be willing to share traditionally "private" information like production schedules and product structures. The participating companies must agree about the extent of their partnering.

Partnering in this context means an agreement between two or more companies to do business together on a long-term basis. The partners will share information and work together in order to create business benefits. Partnering is possible only in those cases where the business between partners makes up a significant part of the company's business. 
Partnering requires also discussion about business ethics and rules. In order to be as effective and efficient as possible the partners should be open towards each other. This means that they should tell each other about future sales promotion campaigns and other significant events. The main problem of this kind of information is the confidentiality of the information and more exactly the information that is left out or not delivered. In a case example when a wholesaler does not have a promotion activity within a time frame of the partner supplier's products the supplier could figure out possible promotion activity with competitors products. This could then lead to hostile activities trying to minimize effects of the competitors possible promotion, and the outcome would be a difficult situation in the partnership between the initial supplier and the wholesaler.

\section{Communication technology}

The co-operation should be built using open systems technology. The idea is that the same communication system can be used by many different partners. A closed system for bilateral communication is not profitable in the long run since it would require a separate system for each partner. The system should use TCP/IP technology, the internet, to be modern. The actual communication can be realized as EDI (Electronic Data Interchange) messaging, but internet is the current de facto standard for open communication.

Heard (1994) points out that the initial goal to improve the performance of the distribution chain is easily cornupted by a strong technical focus. The quick response movement in the US has not been able to improve the performance of suppliers despite the widespread introduction of communications technologies with potential to enhance the speed and accuracy of communication. The reason for failure is that the speed and accuracy of communication has not been utilised to synchronize production with demand. The operating practices of the distribution chain have remained the same.

\section{CONCLUSIONS AND FURTHER ACTIONS}

The consequences of industrial dynamics in demand amplification can not be exaggerated (Schonberger 1990). It has been even reported in fast growing industries that component factories have been established to supply the biased demand. It has become one of the key competitive advantages to have reliable and up-to-date information on the real market situation. This is one reason why the grocery stores and other consumer product retailers are implementing sophisticated technological means to transmit on-line information of front-line consumption, i.e. by linking the cash registers directly with the inventory system, which in turn is connected to the wholesalers information system. Yet, this is not enough as the overall efficiency stems from collaboration not only in information sharing, but also on the physical material flow level. These two flows must be incorporated into one operational system throughout the supply chain.

As the supply chain integration evolves to an operational system, every part of the system is aware of the materials flow and how this will affect their future 
operation. This requires changes in both the technical and organizational structure of the actors in the supply chain. Especially the relationship between supplier and wholesaler requires a new approach. ECR and the category management view addresses into this problem. The customer will in the future dictate how the supply chain will run its whole operation, from category management to efficient replenishment of goods to the retail stores. Category management takes the viewpoint of the retailer and retail chain. The objective is to create distinct manageable groups of products that consumers perceive as interrelated or substitutable and to define the strategic role of the category in the retail echelon (ECR Best Practices Operating Committee, 1995).

The sales information acquired through cash registers offers the food industry a practical way of collecting a base for efficient management of the operation. One elementary requirement is that the retail stores in the target market have a high level of automation. Also the scanned stores have to represent an extensive part of the target market. This is possible in the Finnish market where the market place of five million inhabitants is dominated by three nation-wide wholesalers and over $90 \%$ of the retail stores are equipped with bar code readers connected to the cash registers. The use of sales information requires ways to integrate the information into existing operation control systems.

Using the sales information as a planning base of production and delivery control gives an opportunity to decrease the effects of supply chain dynamics caused by Forrester and Burbidge effects. An accurate information flow enables flexible production control and therefore better delivery performance and lower inventory levels throughout the chain.

\section{REFERENCES}

A.T Kearney (1993) Logistics Excellence in Europe, Study prepared by A.T. Kearney on behalf of the European Logistics Association

Burbidge, J.L. (1989) Production Flow Analysis - For planning Group Technology, Clarendon Press, Oxford

Burbidge, J.L. (1994) The use of period batch control (PBC) in the implosive industries, Production Planning \& Control, 5, No 1, 97-102

ECR Best Practices Operating Committee, (1995) Category Management Report Enhancing Consumer Value in the Grocery Industry, Joint Industry Project on Efficient Consumer Response, USA,

Forrester, J. (1961) Industrial Dynamics, MTT Press Cambridge, Ma

Heard, E. (1994) Quick response: Technology or knowledge?, Industrial Engineering, August, 28-30

Pontin, M. \& Hutter, L. (1995) Colgate-Palmolive: Meeting our customers' needs, Logistics Focus, 3, No 7, 2-5

Quelch, J. \& Kenny, D. (1994) Extend Profits, Not Product Lines, Harvard Business Review, Sep-Oct

Robins, G. (1994) Sailing into ECR's uncharted waters, Stores, 76, No 10, 43-44 
Schonberger, R.J. (1990) Building a Chain of Customers: Linking Business Functions to Create the World Class Company, Free Press, New York,.

Stalk Jr. G., Hout T.M. (1990) Competing Against Time, The Free Press, New York, USA

Towill, D. (1992) Supply Chain Dynamics - the change engineering challenge of the mid 1990s, Proc. Instn. Mech Engrs, No 206, 233-245,

Towill, D. (1996) Time compression and the supply chain - a guided tour, Supply Chain Management, 1, No 1, 15-27

Yohalem, K. (1995) Is the consumer your CEO?, Sporting Goods Business, 28, No2, 30

\section{BIOGRAPHY}

Jonas Slotte is a project manager at the TAI Research Center at Helsinki University of Technology (HUT). He has been involved in several research projects concerning the logistics management and supply chain control in different industries. His special interest is in the management of the extended supply chain in food industry. He has received his Masters exam at HUT and is a Ph.D. student at the Department of Industrial management at HUT.

Hannu Nyman is a project manager at TAI Research Center at HUT. His previous research has involved IT-tools in the control of the logistics chain. The research work has been in both construction and food industry. He received his Masters diploma (Engineering) from HUT and Masters (Economics) from Helsinki School of Economics and Business Administration and is currently a Ph.D. student at the Department of Industrial management at HUT. He is involved in a major project developing the control practices if information in the Finnish food industry.

Jan Holmström is a Docent at the Department of Industrial Management at HUT. He has been involved in various research projects: Developing support systems for Purchasing Logistics in the automotive industry (Saab-Valmet, Adam Opel AG). Developing Purchasing Logistics concept for a manufacturer of circuit boards. Definition of Production Planning and Control requirements in integrated garments manufacture. Development of efficient logistics practices in the Nordic food industry. He has received his Masters degree at the Department of Computing Sciences and the Licentiate and Ph.D. at the Department of Industrial Management at HUT. 